\title{
Improvement of Chest Wall Compliance Immediately Following Subcutaneous Placement of Central Venous Catheter for Decompression of Massive Subcutaneous Emphysema
}

\author{
Erich Burton ${ }^{1}$ and Antine E Stenbit ${ }^{2,3}$
}

1. Department of Emergency Medicine, Prisma Health-Upstate, Greenville, SC, USA; 2. Division of Pulmonary and Critical Care, Department of Internal Medicine, Prisma Health-Upstate, Greenville, SC, USA; 3. University of South Carolina School of Medicine, Greenville, SC, USA

S ubcutaneous emphysema (SE) and pneumomediastinum occur frequently in association with trauma, soft-tissue infection, chest-tube placement, esophageal rupture, cardiothoracic surgery, and positive pressure ventilation. While typically benign and self-limiting, rarely and unpredictably, rapid accumulation of gas can progress to a state of physiologic compromise requiring prompt intervention; a clinical entity known as massive subcutaneous emphysema (MSE). MSE can lead to compartment syndrome, obstructive shock, skin necrosis, large airway compression, pulmonary barotrauma, and patient discomfort. We report the case of a mechanically ventilated 58-year-old woman who developed decreased chest wall compliance and impaired ventilator mechanics from MSE, requiring emergent decompression. While various methods for decompression have been described in the literature, no single method has been established as most effective. Rapid placement of a central venous catheter into the subcutaneous tissue of the anterior chest wall, attached to suction produced dramatic improvement of tissue swelling, ventilatory peak and plateau pressures, and both dynamic and static compliance. We propose a novel approach for decompression that is quick, minimally invasive, readily accessible, and familiar to clinicians practicing in an intensive care unit or emergency department.

\section{Keywords}

Massive subcutaneous emphysema, tension subcutaneous emphysema, subcutaneous emphysema, catheter decompression, pneumomediastinum

Disclosures: Erich Burton and Antine E Stenbit have no financial or non-financial relationships or activities to declare in relation to this article.

Acknowledgments: We wish to thank the following: the patient and her family who have given consent not only for the procedure, but also to have her photographs and the procedure published; all the staff at Prisma Health North Greenville Hospital, Travelers Rest, SC, USA, for their hard work and dedication in taking care of patients with COVID-19; and Dr. Sean Callahan for his critique and assistance in editing.

Review Process: Double-blind peer review.

Compliance with Ethics: Informed consent was received from the patient involved in this case study.

Authorship: The named authors meet the International Committee of Medical Journal Editors (ICMJE) criteria for authorship of this manuscript, take responsibility for the integrity of the work as a whole, and have given final approval for the version to be published.

Access: This article is freely accessible at touchRESPIRATORY.com (C) Touch Medical Media 2020

Received: October 22, 2020

Accepted: November 23, 2020

Published Online: December 23, 2020

Citation: US Respiratory \& Pulmonary Diseases. 2020;5(1):48-51

Corresponding author: Antine E Stenbit, 200 Patewood Dr, Building B Suite 300, Greenville, SC 29615, USA. E: Antine.Stenbit@Prismahealth.org

Support: No funding was received in the publication of this article.
Subcutaneous emphysema (SE) is relatively common in the intensive care unit (ICU) and often a complication of positive pressure ventilation. ${ }^{12}$. While typically self-limiting and benign, life-threatening sequela can develop. Progressive accumulation of subcutaneous air can result in significant compressive forces leading to the development of a rare clinical entity known as massive subcutaneous emphysema (MSE). The hallmark of MSE is characterized by significant compressive forces leading to physiologic compromise requiring urgent or emergent decompression. Depending on the location, this can lead to tissue necrosis, tracheal and vascular compression, decreased chest wall compliance, pulmonary barotrauma, compartment syndrome, and obstructive shock secondary to tension pneumomediastinum or pneumothorax. ${ }^{3-5}$ These patients present an interesting challenge and warrant temporizing measures in the absence of definitive treatment. As MSE can develop in a variety of clinical settings (i.e., intensive care unit, emergency department, operating room, or spontaneously outside of the hospital), prompt recognition and technically simple means of decompression are paramount. We report a case of MSE that resulted in severe soft tissue swelling, decreased dynamic $\left(\mathrm{C}_{\text {dyn }}\right)$ and static $\left(\mathrm{C}_{\text {stat }}\right)$ compliance, and respiratory compromise. This was successfully treated in a novel and practical fashion by placement of a triple-lumen central venous catheter into the subcutaneous tissue of the anterior chest wall.

\section{Case presentation}

A 58-year-old woman without significant co-morbid conditions was transferred for management of SARS-COV-2 (COVID-19) infection, superimposed bacterial pneumonia, and acute hypoxemic respiratory failure, meeting the Berlin criteria for moderate acute respiratory distress 
Figure 1: Triple-lumen catheter attached to wall-suction tubing at distal port

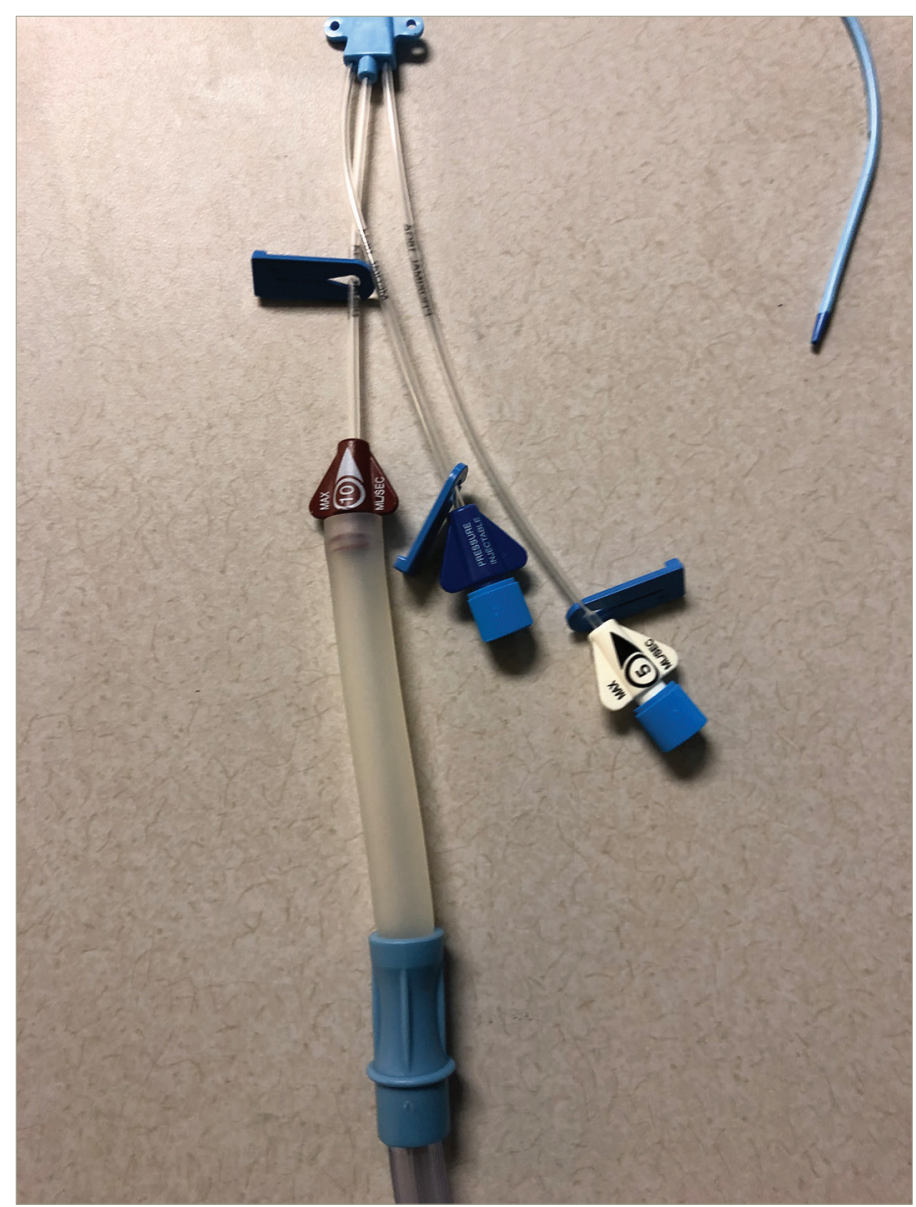

Suction tubing fits snuggly over the top of distal port site and connects to wall-suction via double-ended barbed connector.

syndrome (ARDS) $\left(\mathrm{PaO}_{2} / \mathrm{FiO}_{2}\right.$ ratio $\leq 200$ and positive end expiratory pressure $[\mathrm{PEEP}] \geq 5 \mathrm{~cm} \mathrm{H} \mathrm{H}_{2} \mathrm{O}$ ). ${ }^{6}$ The patient had been mechanically ventilated per ARDSnet protocol prior to transfer, treated with piperacillin/tazobactam, hydroxychloroquine, and zinc, and weaned from epoprostenol while at the outside hospital. Upon arrival, physical examination was notable for crepitus and moderate swelling over anterior right chest and neck. Point of care ultrasound demonstrated bilateral lung sliding and non-plethoric inferior vena cava, suggesting the crepitus was most likely from a pneumomediastinum and not a pneumothorax. The chest $x$-ray demonstrated multifocal bilateral infiltrates, moderate pneumomediastinum with extensive SE, and no evidence of pneumothorax.

The ventilator was on pressure-regulated volume control: respiratory rate 16 , tidal volume $360 \mathrm{cC}$, PEEP $14 \mathrm{~cm} \mathrm{H}_{2} \mathrm{O}$, and $\mathrm{FiO}_{2}$ 60\%. These settings were consistent with ARDSnet protocol. Peak $\left(\mathrm{P}_{\text {peak }}\right)$ and plateau $\left(\mathrm{P}_{\text {plat }}\right)$ pressures were $22-28 \mathrm{~cm} \mathrm{H}_{2} \mathrm{O} . \mathrm{C}_{\text {dyn }}$ and $\mathrm{C}_{\text {stat }}$ were $40-50 \mathrm{~mL} / \mathrm{cm} \mathrm{H}_{2} \mathrm{O}$. Over 24 hours the patient developed worsening SE tracking to the facial tissue planes causing severe periorbital emphysema. PEEP was lowered from $14 \mathrm{~cm}$ to $6 \mathrm{~cm} \mathrm{H} \mathrm{H}_{2} \mathrm{O}$, and $\mathrm{FiO}_{2}$ was increased from 0.6 to 0.8 in an attempt to mitigate the expansion of SE. The following day we were unable to open the patient's eyelids because of profound swelling. Severe hypoxemia precluded further reduction in PEEP or tidal volume. $\mathrm{P}_{\text {peak }}$ and $\mathrm{P}_{\text {plat }}$ remained elevated above $30 \mathrm{~cm} \mathrm{H} \mathrm{H}_{2} \mathrm{O}$ with $\mathrm{C}_{\text {dyn }}$
Figure 2: Chest radiograph
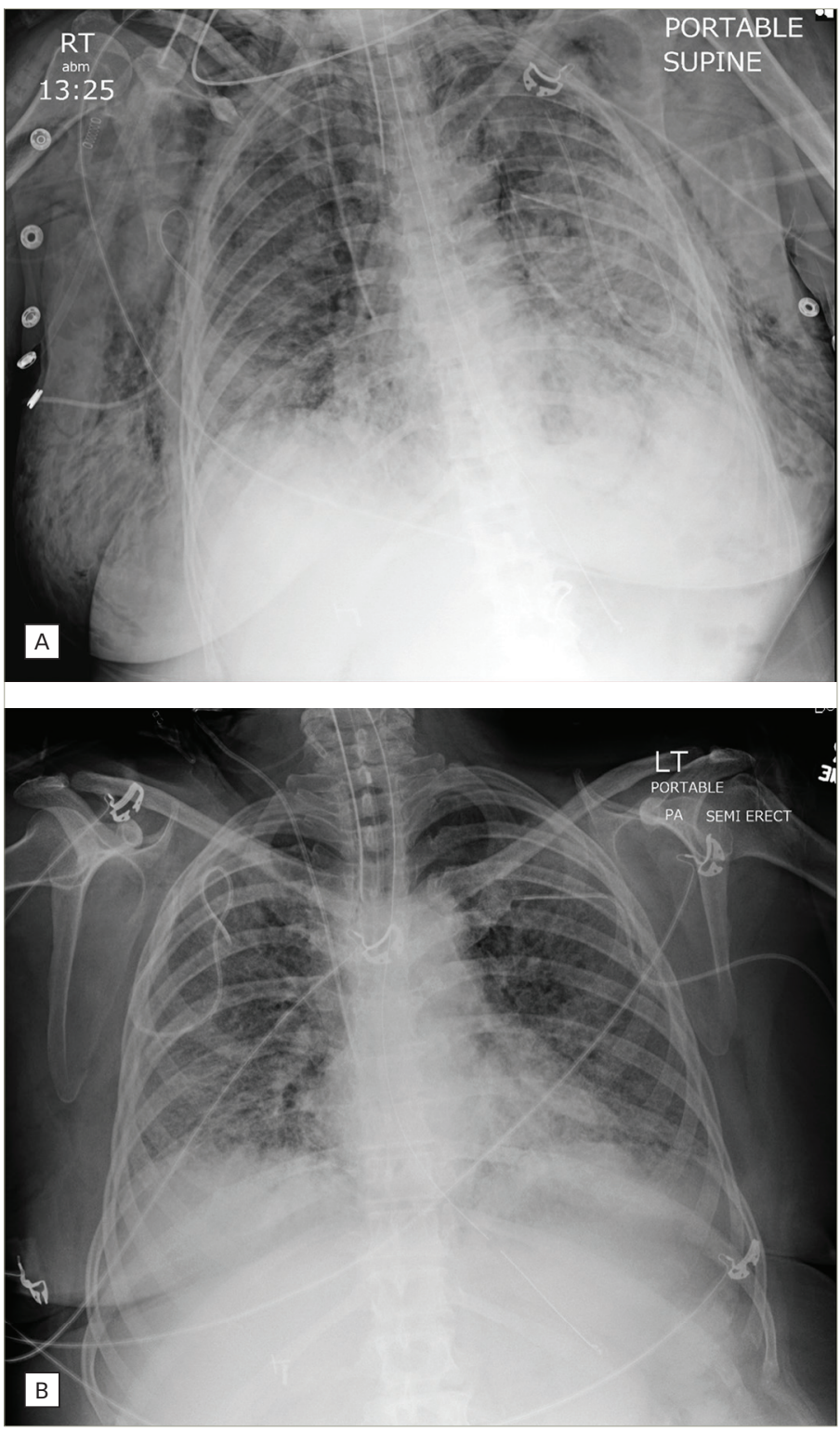

Significant subcutaneous emphysema immediately after placement of second triple-lumen catheter (A), with subsequent radiographic improvement on day 6 (B).

and $\mathrm{C}_{\text {stat }}<25 \mathrm{~mL} / \mathrm{cm} \mathrm{H}_{2} \mathrm{O}$. Air artifact precluded further use of lung point of care ultrasound. Breath sounds remained present bilaterally. After consideration of the patient's clinical status and ventilator data we felt the elevated airway pressures represented a combination of shunt physiology from ARDS and acutely reduced chest wall compliance from MSE. Given persistent worsening and the improbability of resolution while on positive pressure ventilation the decision was made to attempt rapid bedside decompression.

While our facility is an adequately equipped, tertiary care ICU, at the time it was being used for solely treating patients with COVID-19, and did not have surgical consultation immediately available. This clinical context is important as management strategies may differ when caring for critically-ill patients with variable access to consultants. Without previous training in performing open "blow-hole" incisions or mediastinotomy, we favored the novel use of a standard triple-lumen central venous catheter. Prior to 
Figure 3: Day 1 immediately following insertion of the first triple-lumen catheter

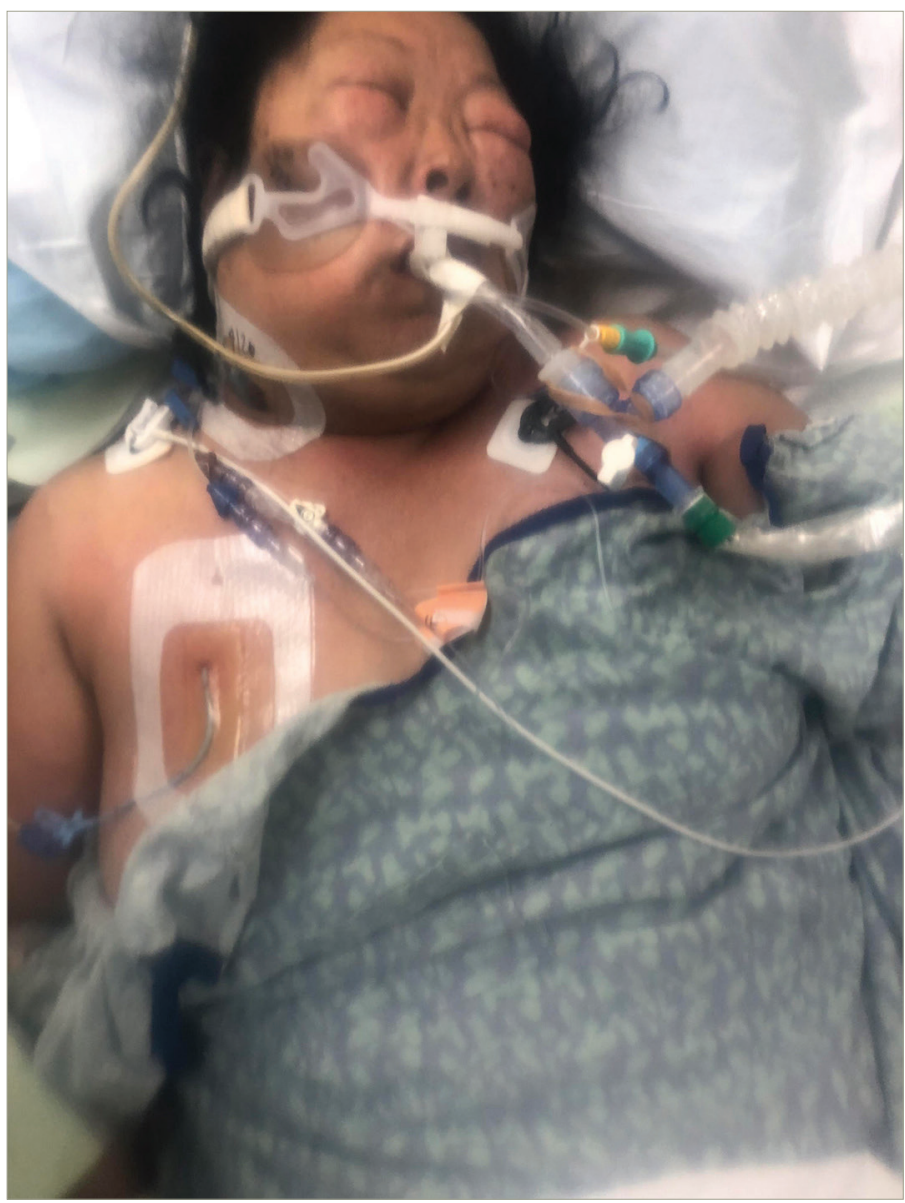

the procedure, consent was obtained from the healthcare proxy. Using Seldinger wire technique, an 18-gauge introducer needle was placed at $<45$ degree angle into the subcutaneous tissue at the third-intercostal space of the mid-clavicular right chest. Air was aspirated upon entering the subcutaneous tissue. A wire was advanced. A 7-French (Fr) triple-lumen central venous catheter was advanced over the guidewire and placed under the skin to $10 \mathrm{~cm}$. The proximal and middle ports were sealed. Air was expressed through the distal port with immediate improvement of tissue swelling. Within 1 hour, $\mathrm{P}_{\text {plat }}$ and $\mathrm{P}_{\text {peak }}$ were persistently $<20-25 \mathrm{~cm} \mathrm{H}_{2} \mathrm{O}$; $\mathrm{C}_{\text {dyn }}$ and $\mathrm{C}_{\text {stat }}$ were $40-50 \mathrm{~mL} / \mathrm{cm} \mathrm{H}_{2} \mathrm{O}$; and $\mathrm{PaO}_{2}$, oxygen saturation, and tidal volumes were improving. The distal port was connected to wall suction via a piece of Lukens specimen container tubing, which was then attached to the male connector present on standard suction connector tubing (Figure 1). The catheter was marked "NOT CENTRAL ACCESS and NOT FOR IV FLUIDS." Soft-tissue swelling and ventilator mechanics continued to improve over the next 24 hours. Approximately 72 hours later, the patient required decompression of the left anterior chest for worsened SE exacerbated by increasing PEEP requirements. The procedure was repeated on the opposite side of the chest with subsequent improvement of above stated parameters. No complications were observed (Figures 2-4).

\section{Discussion}

SE characterizes the accumulation of gas or air within the subcutaneous tissue. Diagnosis is typically made clinically by palpation of crepitus
Figure 4: Day 3 after insertion of the second triple-lumen catheter and significant improvement of right-sided subcutaneous emphysema

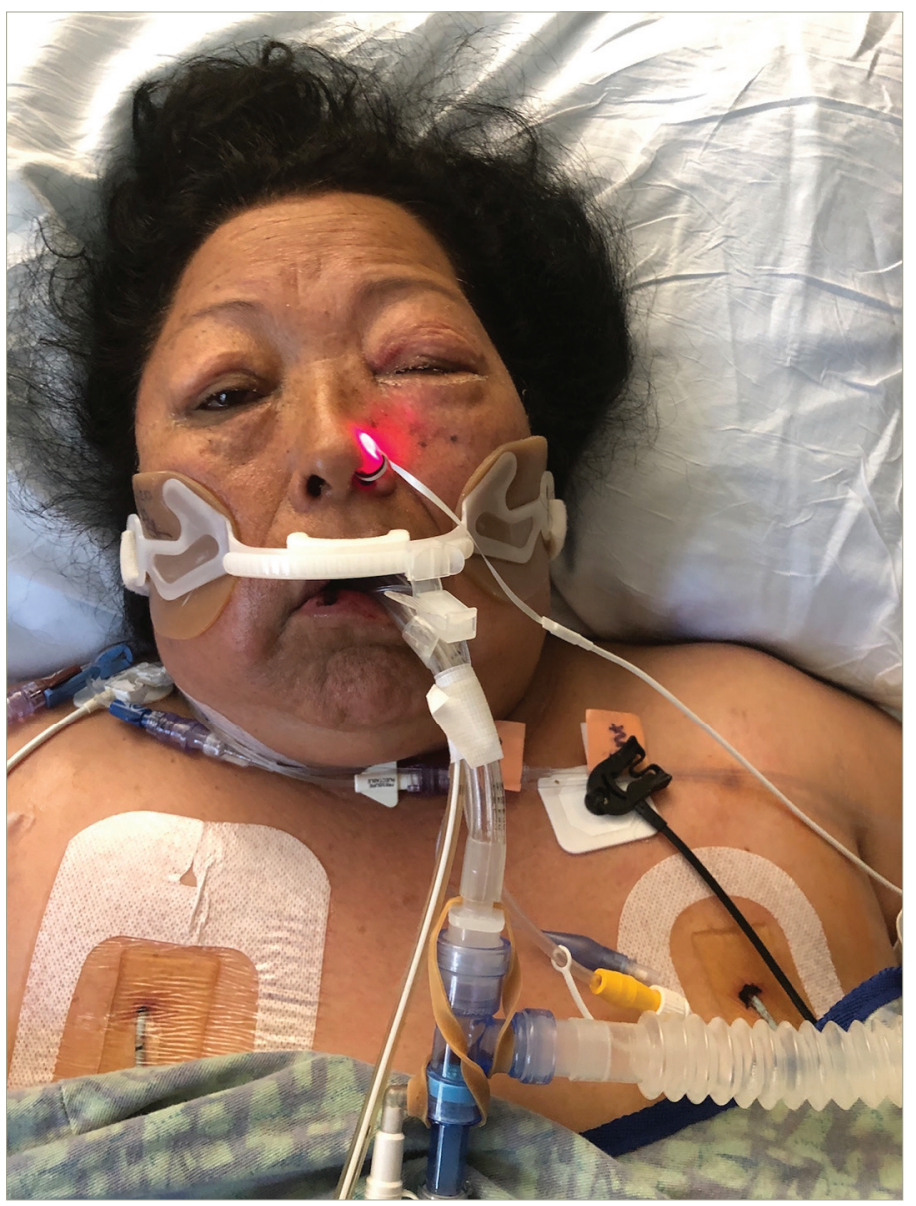

and noticeable tissue swelling. In more subtle cases, imaging (X-ray or computed tomography scan) can assist with diagnosis. The most common etiologies include pneumomediastinum, pneumothorax, soft-tissue infections, traumatic injuries, surgery, and positive pressure ventilation. Less well-known causes include asthma exacerbation; pneumorrhachis; and pulmonary barotrauma associated with MDMA (3,4-methylenedioxymethamphetamine), heroin, and cocaine abuse. $5,7,8$ When SE is a result of positive pressure ventilation, barotrauma-induced alveolar or bleb rupture allows air to escape and dissect the bronchovascular sheaths. Air then enters the perivascular interstitium and travels along the sheaths towards the hilum, where it can pass into the mediastinal, pleural, and/or subcutaneous spaces. ${ }^{9}$

SE most commonly occurs along the fascial plane of the anterior thoracic cavity. As a result of contiguous fascial planes, subcutaneous air in the chest can travel to the retroperitoneum, neck, and face. ${ }^{4}$ Although rare, MSE results when air accumulation and compressive forces become significant enough to cause compartment syndrome, impaired ventilator mechanics, tissue necrosis, or cardiopulmonary instability. Patients undergoing positive pressure ventilation present a particular dilemma as their therapy often worsens MSE yet remains vital and life-sustaining. Without decompression a vicious cycle of elevated $\mathrm{P}_{\text {peak }} / \mathrm{P}_{\text {plat }}$, reduced $\mathrm{C}_{\text {dyn }} / \mathrm{C}_{\text {stat }}$ and worsening hypoxemia will ensue. In our case we believe the elevated $P_{\text {peak }} / P_{\text {plat }}$ 
reduced $\mathrm{C}_{\mathrm{dyn}} / \mathrm{C}_{\text {stat }}$ and worsening hypoxemia were the result of MSE rather than worsening ARDS, as evidenced by the overall improvement immediately following decompression.

When approaching a patient with MSE the importance of identifying the underlying etiology cannot be overstated. In most cases the etiology will inform treatment and intervention (i.e., tube thoracostomy for pneumothorax, surgical debridement for gas gangrene, or discontinuing positive pressure ventilation if tolerated). Historically, there have been countless interventions for subcutaneous decompression. A shared feature among them being the rapid release of trapped air under pressure, with improvement or resolution of symptoms. Methods include incisions or "blow holes" made in the skin, the use of angiocatheters, mediastinal drains, mediastinotomy, subcutaneous placement of 28-Fr thoracostomy tube to water seal, and application of a negative pressure wound vacuum. ${ }^{1-3,5,10,11}$ Our review of the literature found no clear consensus regarding the best technique for rapid decompression of massive SE due to positive pressure ventilation associated pneumomediastinum without pneumothorax. Management appears to be heavily influenced by clinician experience, clinical setting, and underlying etiology. We posit that clinician acumen and time to decompression are more clinically relevant than any one specific technique.

A review of the literature revealed numerous case reports of angiocatheter and subcutaneous drain placement for decompression, but did not yield previously reported cases using a triple-lumen central venous catheter. The technique we describe in this case was influenced by previously reported methods and offers a few subtle advantages: longer catheter length, multiple sites for suction, confirmation via real-time aspiration, and familiarity to critical care practitioners. Central venous catheters allow for better securement due to longer length and ease of suture securement when compared with a standard angiocatheter. While we did not use multiple ports in our case, multi-lumen access should be considered a potential benefit as it can be used for additional suction or back-up access if the primary lumen fails. Variability in angiocatheter design makes it difficult to predict luer lock compatibility, as certain angiocatheters are only compatible with a 10-cc syringe luer lock once the catheter has been removed from the needle; this makes needle aspiration for confirmation, impossible. Our method uses an 18-gauge introducer needle with a small volume of sterile saline in a $10-c c$ syringe that can be used to confirm placement within emphysematous tissue upon aspiration of air bubbles. As placement of a triple-lumen central venous catheter utilizes skills already possessed by critical care practitioners there is the potential for quicker placement and fewer complications.

MSE can develop in a variety of settings and necessitates prompt recognition and decompression utilizing readily available equipment. The technique we describe here resulted in rapid improvement and reversal of a life-threatening condition. While various suitable methods exist, the repurposing of a well-familiarized instrument could lead to improvement of the most important metrics: time to decompression and hemodynamic stabilization. Our technique is an easy, safe, practical, and effective method for treating MSE. 口
1. Maunder RJ, Pierson DJ, Hudson LD. Subcutaneous and mediastinal emphysema. Pathophysiology, diagnosis, and management. Arch Intern Med. 1984;144:1447-53.

2. Robinson B. Rapid resolution of severe subcutaneous emphysema with simple percutaneous angiocatheter decompression. J Surg Case Rep. 2018;2018:rjy173.

3. Kubik T, Niewiński G, Wojtaszek M, et al. The skin incisions (blow holes) for treatment of massive subcutaneous emphysema Anestezjol Intens Ter. 2011;43:93-7.

4. Kouritas VK, Papagiannopoulos K, Lazaridis G, et al. Pneumomediastinum. J Thorac Dis. 2015;7(Suppl. 1):S44-9.
5. Aghajanzadeh M, Dehnadi A, Ebrahimi H, et al. Classification and management of subcutaneous emphysema: a 10-year experience. Indian J Surg. 2015;77(Suppl. 2):673-7.

6. Ranieri VM, Rubenfeld GD, Thompson BT. The ARDS Definition Task Force. Acute respiratory distress syndrome: the Berlin definition. JAMA. 2012;307:2526-33.

7. Wurcel AG, Merchant EA, Clark RP, Stone DR. Emerging and underrecognized complications of illicit drug use. Clin Infect Dis. 2015;61:1840-9.

8. Liu YW, Kao CN, Wang YM. Pneumorrhachis: an under-recognized entity correlates with severity of spontaneous pneumomediastinum.
JThorac Dis. 2018:10: E149-51.

9. Gesundheit B, Preminger A, Harito B, et al. Pneumomediastinum and subcutaneous emphysema in an 18-month-old child. J Pediatr. 2002;141:116-20

10. Taylor BC, McGowan S. Use of closed incision negative pressure therapy for massive subcutaneous emphysema. Cureus. 2020:12:e7399.

11. Kelly MC, MCGuigan JA, Allen RW. Relief of tension subcutaneous emphysema using a large bore subcutaneous drain. Anaesthesia. 1995;50:1077-9. 